\title{
Photometric colors of late-type giants: theory versus observations
}

\author{
A. Kučinskas ${ }^{12}$, P.H. Hauschildt ${ }^{3}$, H.-G. Ludwig ${ }^{4}$, I. Brott ${ }^{3}$, \\ V. Vansevičius ${ }^{5}$, L. Lindegren ${ }^{6}$, T. Tanabé ${ }^{7}$ and F. Allard ${ }^{8}$ \\ ${ }^{1}$ National Astronomical Observatory of Japan, Mitaka, Tokyo 181-8588, Japan \\ email: arunas.kucinskas@nao.ac.jp \\ ${ }^{2}$ Institute of Theoretical Physics and Astronomy, Goštauto 12, Vilnius 01108, Lithuania \\ email: ak@itpa.lt \\ ${ }^{3}$ Hamburger Sternwarte, Gojenbergsweg 112, 21029 Hamburg, Germany \\ ${ }^{4}$ GEPI - CIFIST, Observatoire de Paris-Meudon, 5 place Jules Janssen, \\ 92195 Meudon Cedex, France \\ ${ }^{5}$ Institute of Physics, Savanoriu 231, Vilnius 02300, Lithuania \\ ${ }^{6}$ Lund Observatory, Lund University, Box 43, SE-221 00 Lund, Sweden \\ ${ }^{7}$ Institute of Astronomy, The University of Tokyo, Mitaka, Tokyo, 181-0015, Japan \\ ${ }^{8}$ CRAL, École Normale Supérieure, Lyon, Cedex 07, 69364 France
}

Late-type giants (i.e., stars on the red and asymptotic giant branches, RGB/AGB, respectively) are dominant contributors to the overall spectral appearance of intermediate age and old stellar populations, especially in the red/near-infrared part of the spectrum. Being intrinsically bright, they are well suited for probing distant/obscured populations, especially those that can not be studied with their fainter members, like main sequence turn-off stars or subgiants. Late-type giants and supergiants will be the only stellar types accessible in intermediate age and old populations beyond the distances of several Mpc with the future 30-50 m class extremely large telescopes (Olsen et al. 2003). Indeed, proper understanding of their observable properties by means of theoretical models is of key importance for studying the evolution of stellar populations and their host galaxies.

To assess the current status in the theoretical modeling of the spectral properties of late-type giants, we provide a comparison of synthetic photometric colors of late-type giants (calculated with PHOENIX, MARCS and ATLAS model atmospheres) with observations, at $[\mathrm{M} / \mathrm{H}]=0.0$ and -2.0 (Fig. 1). Overall, there is a good agreement between synthetic colors and observations, and synthetic colors and published $T_{\text {eff }}-$ color relations, both at $[\mathrm{M} / \mathrm{H}]=0.0$ and -2.0 . Deviations from the observed trends in $T_{\text {eff }}-$ color planes are generally within $\pm 150 \mathrm{~K}$ (or less) in the effective temperature range of $T_{\text {eff }}=3500-$ 4800 K. Synthetic colors calculated with different stellar atmosphere models typically agree to $\sim 100 \mathrm{~K}$, within a large range of effective temperatures and gravities. Some discrepancies are seen in the $T_{\text {eff }}-(B-V)$ plane below $T_{\text {eff }} \sim 3800 \mathrm{~K}$ at $[\mathrm{M} / \mathrm{H}]=0.0$, due to difficulties in reproducing the 'turn-off' to the bluer colors which is seen in the observed data at $T_{\text {eff }} \sim 3600 \mathrm{~K}$. Note that at $[\mathrm{M} / \mathrm{H}]=-2.0$ effective temperatures given by the scale of Alonso et al. (1999, A99) are generally lower than those resulting from other $T_{\text {eff }}$-color relations based both on observed and synthetic colors. This is clearly seen in all $T_{\text {eff }}$-color planes, with an average offset of $\sim 130 \mathrm{~K}$.

Obviously, reasonably good agreement can be achieved between theoretical predictions and observed properties of late-type giants at the level of about $\pm 150 \mathrm{~K}$; however, systematic differences in individual $T_{\text {eff }}$-color planes may easily reach (or even exceed) $\pm 100 \mathrm{~K}$. While this may point towards an interplay of various factors to be clarified in a 

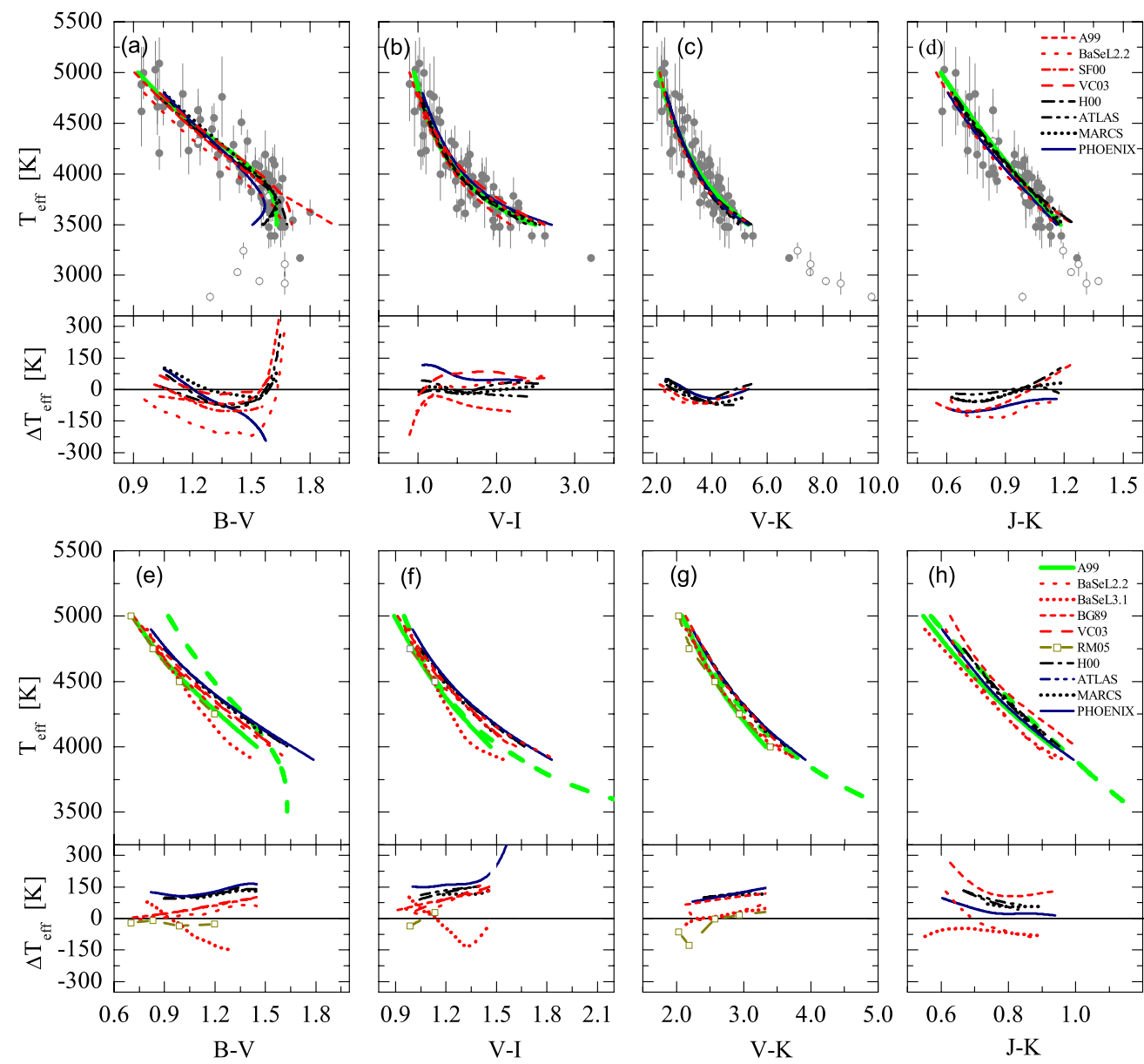

Figure 1. Empirical and theoretical $T_{\text {eff }}$-color relations for late-type giants in different $T_{\text {eff }}-$ color planes, at $[\mathrm{M} / \mathrm{H}]=0.0$ (panels $\mathrm{a}-\mathrm{d})$ and $[\mathrm{M} / \mathrm{H}]=-2.0(\mathrm{e}-\mathrm{h})$. Filled circles are late-type giants from the Solar neighborhood, variable stars are highlighted as open circles (in both cases effective temperatures are derived from interferometry). Thick solid line is a best-fit to the data at $[\mathrm{M} / \mathrm{H}]=0.0$ (panels a-d; also shown as thick dashed line in panels $\mathrm{e}-\mathrm{h}$ ); thick lines in panels $\mathrm{e}-\mathrm{h}$ are $T_{\text {eff }}-$ color relations of A99 at $[\mathrm{M} / \mathrm{H}]=-2.0$. Several existing $T_{\text {eff }}$-color relations are shown as well (BaSeL 3.1: Westera et al. 2002, A\&A, 381, 524; BG89: Bell \& Gustafsson 1989, MNRAS, 236, 653; RM05: Ramírez \& Meléndez 2005, ApJ, 626, 465; see Kučinskas et al. 2005 for other abbreviations), together with semi-empirical scales constructed using synthetic colors of PHOENIX, MARCS and ATLAS. The bottom panels in each figure show the difference between various $T_{\text {eff }}$-color relations and either the best-fit scale $([\mathrm{M} / \mathrm{H}]=0.0)$ or $T_{\text {eff }}-$ color relations of A99 $([\mathrm{M} / \mathrm{H}]=-2.0)$, in a given $T_{\text {eff }}$-color plane $\left(\Delta T_{\text {eff }}=T_{\text {eff }}^{\text {other }}-T_{\text {eff }}^{\text {bestfit } / \text { A99 }}\right)$.

dedicated analysis (inadequacies in current theoretical models, intrinsic differences in the atmospheres of individual stars), $\pm 100 \mathrm{~K}$ may represent a reasonable lower error margin in $T_{\text {eff }}$ of late-type giants obtainable with currently available stellar atmosphere models.

\section{References}

Alonso, A., Arribas, S. \& Martinez-Roger, C. 1999, A\&AS 140, 261 (A99)

Kučinskas, A., Hauschildt, P.H., Ludwig, H.-G. et al. 2005, A\&A 442, 281

Olsen, K.A., Blum, R.D. \& Rigaut, F. 2003, AJ 126, 452 